\title{
Modulation of the gravitational waveform by the effect of radiation reaction
}

\author{
Yasushi Mino* \\ Mail Code 130-33, California Institute of Technology, Pasadena, California 91125, USA
}

(Received 19 November 2007; published 7 February 2008)

\begin{abstract}
When we calculate gravitational waveforms from extreme-mass-ratio inspirals by metric perturbation, it is a common strategy to use the adiabatic approximation. Under that approximation, we first calculate the linear metric perturbation induced by geodesics orbiting a black hole, then we calculate the adiabatic evolution of the parameters of geodesics due to the radiation reaction effect through the calculation of the self-force. This procedure is considered to be reasonable, however, there is no direct proof that it can actually produce the correct waveform we would observe. In this paper, we study the formal expression of the second order metric perturbation and show that it can be expressed as the linear metric perturbation modulated by the adiabatic evolution of the geodesic. This evidence supports the assumption that the adiabatic approximation can produce the correct waveform, and that the adiabatic expansion we propose in Ref. [Y. Mino, Prog. Theor. Phys. 115, 43 (2006); Y. MinoProg. Theor. Phys.113, 733 (2005); Y. Mino and R. Price (unpublished).] is an appropriate perturbation expansion for studying the radiation reaction effect on the gravitational waveform.
\end{abstract}

DOI: 10.1103/PhysRevD.77.044008

PACS numbers: 04.30.Db, 04.25.Nx

\section{INTRODUCTION}

We study the gravitational waveforms from particles moving around Kerr black holes by using a metric perturbation method. There is an established method for calculating these waveforms from the linear metric perturbation of Kerr black holes. By the consistency of the Einstein equation, the source stress-energy tensor of the linear metric perturbation must satisfy the conservation law with respect to the background. As a result, the source of the linear metric perturbation moves along a geodesic of the background Kerr metric. Because of the integrability of the geodesic equation for the Kerr metric, its bound solutions are stable and have periodic features. To understand some features of gravitational waves from such a stable system, we develop a technique of formal calculation [1]. The advantage of this technique is that, one can easily grasp some key features of the waveform without a complicated calculation, and it helps us to construct the strategy for an explicit calculation. Using this technique, we find that the waveform from the linear metric perturbation has a periodic feature [1] as we review in Sec. II. Based on our understanding of this feature, a present numerical code is trying to identify which of the gravitational wave modes are strong enough to be observed by gravitational wave detectors [2].

Because gravitational waves carry away energy and angular momentum, the system must have a dissipative evolution. This should change the periodic features of the gravitational waveform that we know from the linear metric perturbation. New features must be seen in the second order metric perturbation because the source term must include this dissipative effect. The goal of this paper is to find these new features. That is, the goal is to find how the

*mino@tapir.caltech.edu wave amplitude and phase are modulated by gravitational radiation reaction. However, a serious calculation of the second order metric perturbation still has a lot of technical difficulties. We therefore extend the technique of formal calculation we developed to study the linear metric perturbation. We expect that the result of this formal calculation will be useful in future explicit calculations of the second order metric perturbation.

This problem has attracted the attention of the gravity community [3] because of its relevance to gravitational wave detectors, especially LISA. Among the primary targets for LISA are extreme-mass ratio inspirals (EMRIs), the inspiralling binary systems of supermassive black holes (with mass $\sim 10^{5}-10^{9} M_{\odot}$ ), and stellar mass compact objects (with mass $\sim 1-100 M_{\odot}$ ). Because of the extreme mass ratios, the metric perturbation of the black hole is effective for studying the dynamics of the system; we use the Kerr geometry of the supermassive black hole as a background, and approximate the compact object as a point particle. The linear metric perturbation may predict the waveform at an instant. However, LISA will detect gravitational waves for several years. This time scale is comparable to the radiation reaction time scale of EMRIs and is essential to consider the dissipation effect of the gravitational radiation reaction because the effect will accumulate during the observation time.

Most of the relevant investigations made so far discuss the calculation of the self-force [4] which needs regularization [5]. The underlying idea for such calculations is that: (1) At each instant, the orbit is approximated by a geodesic since, due to the extreme mass ratio, gravitational radiation reaction is a small effect. (2) As the effect of radiation reaction accumulates over time, the orbit changes from one geodesic to another. (3) These changes can be deduced from the self-force. This idea is usually referred as the "adiabatic" approximation. This calculation strategy 
has some theoretical problems. Since the self-force is gauge dependent, we may not be able to make a unique prediction about the orbital evolution. In Ref. [6], we showed that, for times over which the standard metric perturbation expansion is valid, the self-force can be arbitrarily adjusted via gauge changes. As an extreme example, we showed that there is a gauge transformation which completely eliminates the self-force.

Do we still need to calculate the self-force? In Ref. [6], we argue that the answer may be yes. Under a certain gauge condition, the self-force may include the correct radiation reaction effect. With this gauge condition, the calculation by the adiabatic approximation will give us the correct prediction of the gravitational waveform. The present paper discusses the self-force under that "physically reasonable" class of gauge conditions proposed in Refs. [1,6] in the context of the second order metric perturbation. We will focus on gauge invariant quantities. The basic idea is that, because the gravitational waveform is observable, the features we can see from the waveform have invariant meanings. We will show that the self-force correctly describes the radiation reaction effect through the second order metric perturbation.

The field equations for the linear and second order metric perturbations are derived as follows. We expand the metric in a small parameter $\epsilon$

$$
g_{\mu \nu}=g_{\mu \nu}^{(0)}+\epsilon g_{\mu \nu}^{(1)}+\epsilon^{2} g_{\mu \nu}^{(2)}+O\left(\epsilon^{3}\right),
$$

where $g_{\mu \nu}^{(0)}$ is the background metric. We insert the $\epsilon$-expansion of the metric into the Einstein tensor and expand it in powers of $\epsilon$. We formally obtain

$$
\begin{aligned}
G^{\mu \nu}= & \epsilon G^{[1] \mu \nu}\left[\mathbf{g}^{(1)}\right]+\epsilon^{2}\left\{G^{[1] \mu \nu}\left[\mathbf{g}^{(2)}\right]\right. \\
& \left.+G^{[2] \mu \nu}\left[\mathbf{g}^{(1)}, \mathbf{g}^{(1)}\right]\right\}+O\left(\epsilon^{3}\right),
\end{aligned}
$$

where $G^{[1] \mu \nu}[\mathbf{h}]$ is linear in $h_{\mu \nu}$ and $G^{[2] \mu \nu}\left[\mathbf{h}^{(1)}, \mathbf{h}^{(2)}\right]$ is bilinear in $h^{[1] \mu \nu}$ and $h_{\mu \nu}^{(2)}$. By similarity expanding the source term

$$
T^{\mu \nu}=\epsilon T^{[1] \mu \nu}+\epsilon^{2} T^{[2] \mu \nu}+O\left(\epsilon^{3}\right),
$$

we can formally write the perturbed Einstein equations to second order $\epsilon$

$$
\begin{gathered}
G^{[1] \mu \nu}\left[\mathbf{g}^{(1)}\right]=8 \pi T^{[1] \mu \nu}, \\
G^{[1] \mu \nu}\left[\mathbf{g}^{(2)}\right]=-G^{[2] \mu \nu}\left[\mathbf{g}^{(1)}, \mathbf{g}^{(1)}\right]+8 \pi T^{[2] \mu \nu} .
\end{gathered}
$$

In Sec. II, we review the derivation of (1.4) [1]. Because of the consistency of the Einstein equation, the source of the linear metric perturbation, $T^{[1] \mu \nu}$, must be a geodesic. Because we are interested in a bound geodesic, bound geodesics are triperiodic, and we will see that the linear metric perturbation induced by it is as well. Before discussing the second order equation (1.5), we make a formal argument about the self-force and the orbital evolution due to this effect in Sec. III because those effects should be included in the source of the second order equation, $T^{[2] \mu \nu}$. In Sec. IV, we discuss the formal analysis of the second order Einstein equation (1.5). The second order metric perturbation is induced by the quadratic term of the linear metric perturbation, and by the second order source term. We emphasize these two effects lead to substantially different features of the second order metric perturbation. Sec. V concludes our result.

Our background geometry will be a Kerr black hole with mass $M$ and spin parameter $a$. We will use a point particle with mass $\mu$ as the source of the metric perturbation. Throughout this paper, we use Boyer-Lindquist coordinates, $\{t, r, \theta, \phi\}=\left\{x^{\alpha}\right\}$. We adopt the geometrized units which are defined such that $G=c=1$.

\section{LINEAR METRIC PERTURBATION}

In this section, we review the periodic feature of the linear metric perturbation. It is well known that, when the background is a vacuum solution, the source term of the linear metric perturbation must be conserved with respect to the background. Since we use a point mass source, this means that its world line is a geodesic of the background geometry. Because we are interested in gravitational waves from binaries in the inspiralling phase, we only consider bound geodesics.

We denote the orbital coordinates of the geodesic by $\left\{\bar{x}^{\alpha}\right\}$ and the 4-velocity by $\bar{v}^{\alpha}:=d \bar{x}^{\alpha} / d \tau$, where $\tau$ is the proper time. The geodesic equation for a Kerr black hole has three nontrivial constants of motion: the energy $E=$ $\mu \eta_{\alpha}^{E} \bar{v}^{\alpha}$, the $z$-component of angular momentum $L=$ $\mu \eta_{\alpha}^{L} \bar{v}^{\alpha}$, and the Carter constant $C=(\mu / 2) \eta_{\alpha \beta} \bar{v}^{\alpha} \bar{v}^{\beta}$. Here $\eta_{\alpha}^{E}$ and $\eta_{\alpha}^{L}$ are temporal and rotational Killing vectors, respectively, and $\eta_{\alpha \beta}$ is the Killing tensor. Hereafter we collectively denote these constants by $\mathcal{E}^{a}$. The geodesic equations can then be formally written as

$$
\begin{aligned}
&\left(\frac{d \bar{r}}{d \lambda}\right)^{2}= R\left(\bar{r} ; \mathcal{E}^{a}\right), \quad\left(\frac{d \bar{\theta}}{d \lambda}\right)^{2}=\Theta\left(\bar{\theta} ; \mathcal{E}^{a}\right), \\
& \frac{d \bar{t}}{d \lambda}=T_{r}\left(\bar{r} ; \mathcal{E}^{a}\right)+T_{\theta}\left(\bar{\theta} ; \mathcal{E}^{a}\right), \\
& \frac{d \bar{\phi}}{d \lambda}=\Phi_{r}\left(\bar{r} ; \mathcal{E}^{a}\right)+\Phi_{\theta}\left(\bar{\theta} ; \mathcal{E}^{a}\right),
\end{aligned}
$$

where $\lambda$ is the affine parameter of the orbit related with the proper time by $\lambda=\int d \tau /\left(\bar{r}^{2}+a^{2} \cos ^{2} \bar{\theta}\right)$. For bound geodesics, the $r$-motion and the $\theta$-motion are periodic and one can write them as a discrete Fourier series. We formally write

$$
\begin{array}{r}
\bar{r}=\sum_{k} r_{k} e^{i k \chi_{r}}, \quad \chi_{r}=\Upsilon_{r}\left(\lambda-\lambda^{r}\right), \\
\bar{\theta}=\sum_{l} \theta_{l} e^{i l \chi_{\theta}}, \quad \chi_{\theta}=\Upsilon_{\theta}\left(\lambda-\lambda^{\theta}\right),
\end{array}
$$


where $\lambda^{r}$ and $\lambda^{\theta}$ are constants for integration. The expansion coefficients, $r_{k}$ and $\theta_{l}$, and the effective frequencies, $\Upsilon_{r}$ and $\Upsilon_{\theta}$, are functions of the constants of motion $\mathcal{E}^{a}$. Using (2.3) and (2.4), we can formally integrate (2.2) as

$$
\begin{gathered}
\bar{t}=\chi_{t}+\sum_{k} t_{k}^{r} e^{i k \chi_{r}}+\sum_{l} t_{l}^{\theta} e^{i l \chi_{\theta}}, \quad \chi_{t}=\Upsilon_{t}\left(\lambda-\lambda^{t}\right), \\
\bar{\phi}=\chi_{\phi}+\sum_{k} \phi_{k}^{r} e^{i k \chi_{r}}+\sum_{l} \phi_{l}^{\theta} e^{i \chi_{\theta}} \\
\chi_{\phi}=\Upsilon_{\phi}\left(\lambda-\lambda^{\phi}\right),
\end{gathered}
$$

where $\lambda^{t}$ and $\lambda^{\phi}$ are constants of integration. The expansion coefficients, $t_{k}^{r}, t_{k}^{\theta}, \phi_{k}^{r}$, and $\phi_{l}^{\theta}$, and the effective frequencies, $Y_{t}$ and $Y_{\phi}$, are functions of the constants of motion $\mathcal{E}^{a}$. In summary, one can specify a geodesic by three constants of motion $\mathcal{E}^{a}$ and four constants of integration $\lambda^{\alpha}$. Because one is free to choose the zero of the affine parameter $\lambda$, one of four integral constants $\lambda^{\alpha}$ is not physically significant, and the gravitational waveform induced by the geodesic depends only on three differences of the four integral constants [See (2.14).].

Under a certain gauge condition, one can define a tensor Green's function for the linear Einstein equation (1.4). Because the background is stationary and axisymmetric, this tensor Green's function has the form

$$
G_{\alpha \beta \mu \nu}\left(x, x^{\prime}\right)=G_{\alpha \beta \mu \nu}\left(t-t^{\prime}, \phi-\phi^{\prime} ; r, r^{\prime}, \theta, \theta^{\prime}\right) .
$$

For the linear metric perturbation, the source term is described by the stress-energy tensor of a point particle [4] written as

$$
T^{[1] \mu \nu}=\mu \int d \tau \bar{v}^{\mu} \overline{\boldsymbol{v}}^{\nu} \frac{\delta(x-\bar{x}(\tau))}{\sqrt{-\left|g^{[0] \mid}\right|}},
$$

where $\left|g^{[0]}\right|$ is the determinant of the background metric. The corresponding linear metric perturbation is given by

$$
g_{\alpha \beta}^{[1]}(x)=8 \pi \mu \int d \lambda\left(\frac{d \tau}{d \lambda}\right) G_{\alpha \beta \mu \nu}(x, \bar{x}(\lambda)) \bar{v}^{\mu} \overline{\boldsymbol{v}}^{\nu}
$$

Using the formal expression for the geodesic, (2.3), (2.4), (2.5), and (2.6), we obtain the following formal expression for this linear metric perturbation

$$
\begin{gathered}
g_{\alpha \beta}^{[1]}(x)=\sum_{k, l, m} e^{-i \omega_{(k, l, m)} t+i m \phi} g_{\alpha \beta(k, l, m)}^{[1]}(r, \theta) \\
\times e^{i\left(k \omega_{r} t^{r}+l \omega_{\theta} t^{\theta}+m \omega_{\phi} t^{\phi}\right)}, \\
\omega_{(k, l, m)}=k \omega_{r}+l \omega_{\theta}+m \omega_{\phi}, \quad \omega_{r}=\frac{\Upsilon_{r}}{\Upsilon_{t}}, \\
\omega_{\theta}=\frac{\Upsilon_{\theta}}{\Upsilon_{t}}, \quad \omega_{\phi}=\frac{\Upsilon_{\phi}}{\Upsilon_{t}}, \\
t^{r}=\Upsilon_{t}\left(\lambda^{r}-\lambda^{t}\right), \quad t^{\theta}=\Upsilon_{t}\left(\lambda^{\theta}-\lambda^{t}\right), \\
t^{\phi}=\Upsilon_{t}\left(\lambda^{\phi}-\lambda^{t}\right),
\end{gathered}
$$

where the expansion coefficients, $g_{\alpha \beta(k, l, m)}^{[1]}(r, \theta)$, depend only on the constants of motion, $\mathcal{E}^{a}$, and are independent of $\lambda^{r}, \lambda^{\theta}, \lambda^{t}$, and $\lambda^{\phi}$.

The Green's function (2.7) can be obtained under the Lorenz gauge or the Harmonic gauge. However, it can be defined under a larger class of gauge conditions which we call by the "physically reasonable class." Its definition can be understood by considering the residual gauge transformation. By the gauge transformation $x^{\alpha} \rightarrow x^{\alpha}+\xi^{\alpha}$ with the gauge field of the form

$$
\xi^{\alpha}(x)=\sum_{k, l, m} e^{-i \omega_{(k, l, m)} t+i m \phi} \xi_{(k, l, m)}^{\alpha}(r, \theta) e^{i\left(k \omega_{r} t^{r}+l \omega_{\theta} t^{\theta}+m \omega_{\phi} t^{\phi}\right)},
$$

the expansion coefficients $h_{\alpha \beta(k, l, m)}(r, \theta)$ are transformed, but, the formal expression of the linear metric perturbation (2.10) is invariant.

The main features of the gravitational waveforms can be read off from the formal expression (2.10). For any distant observer at $r \rightarrow \infty$ and a specific angular position, the waveform can be written as

$$
h(t)=\sum_{k, l, m} h_{(k, l, m)} e^{-i k \omega_{r}\left(t-t^{r}\right)-i l \omega_{\theta}\left(t-t^{\theta}\right)-i m \omega_{\phi}\left(t-t^{\phi}\right)} .
$$

Because the characteristic frequencies of waves $\omega_{r}, \omega_{\theta}$, and $\omega_{\phi}$ are all associated with geodesics, we may conclude that the waveform (2.14) does not reflect dissipative effects occurring on the radiation reaction time scale. This is to be expected since the source of the linear metric perturbation moves on a stable geodesic.

\section{SELF-FORCE AND THE ORBITAL EVOLUTION}

Because the gravitational wave part of the linear metric perturbation carries away energy, the orbit of the particle deviates away from a geodesic. The effect of the orbital deviation is described by the so-called self-force, that is both induced by and acting on the particle itself. Because the metric perturbation induced by the particle diverges along the orbit, a regularization prescription is necessary to obtain the finite self-force [4]. Up to leading order in the particle's mass, we developed the regularization prescription by the technique of the matched asymptotic expansion. The final result is now called the MiSaTaQuWa self-force [4], and is formally written as

$$
\frac{D}{d \tau} \boldsymbol{v}^{\alpha}:=\mu f^{\alpha}(\tau)=\lim _{x \rightarrow \bar{x}(\tau)} \mu f^{\alpha}\left[\mathbf{g}^{[1]}-\mathbf{g}^{[1] \operatorname{sing}}\right](x),
$$

where the bare term $\mathbf{g}^{[1]}$ represents the full linear metric perturbation induced by the point particle, and the counter term $\mathbf{g}^{[1] \text { sing }}$ is the singular part of the linear metric perturbation to be subtracted for regularization. $f^{\alpha}[]$ is a derivative operator for the self-force. Because both terms $\mathbf{g}^{[1]}$ and $\mathbf{g}^{[1] \text { sing }}$ are divergent along the geodesic, it is necessary to 
evaluate them at a field point $x^{\alpha}$, not at the geodesic. After the subtraction, $\mathbf{g}^{[1]}-\mathbf{g}^{[1] \operatorname{sing}}$ becomes regular along the geodesic, and we may take the limit $x^{\alpha} \rightarrow \bar{x}^{\alpha}$ to obtain the finite self-force.

One can use the formal expression (2.10) to evaluate the bare term $\mathbf{g}^{[1]}$. The formal expression (2.10) is also applicable for the counter term $\mathbf{g}^{[1] \mathrm{sing}}$ because one can derive the Green's function for the counter term in the same form (2.7) as we discussed in Ref. [1]. After some formal calculations, the self-force can be written as

$$
\mu f^{\alpha}=\sum_{k, l} \mu f_{k, l}^{\alpha} e^{i k \chi_{r}+i l \chi_{\theta}}
$$

It is crucial to observe that the orbital equations (2.1) and (2.2) are still true for time dependent $\mathcal{E}^{a}$, and we will use these equations to calculate the orbital evolution due to the self-force. Quantities that evolve due to the self-force are denoted with tilde. The evolution equations for $\tilde{\mathcal{E}}^{a}$ are then

$$
\begin{gathered}
\frac{d}{d \lambda} \tilde{E}=\left(\frac{d \tau}{d \lambda}\right) \mu^{2} \eta_{\alpha}^{E} f^{\alpha}, \quad \frac{d}{d \lambda} \tilde{L}=\left(\frac{d \tau}{d \lambda}\right) \mu^{2} \eta_{\alpha}^{L} f^{\alpha}, \\
\frac{d}{d \lambda} \tilde{C}=\left(\frac{d \tau}{d \lambda}\right) \mu^{2} \eta_{\alpha \beta} \bar{v}^{\alpha} f^{\beta} .
\end{gathered}
$$

If each of the $\tilde{\mathcal{E}}^{a}$ is expanded in a manner similar to (3.2). These equations can be formally integrated to give

$$
\begin{gathered}
\tilde{E}=E_{0}+\langle\dot{E}\rangle \lambda+\sum_{k, l} E_{k, l} e^{i k \chi_{r}+i l \chi_{\theta},} \\
\tilde{L}=L_{0}+\langle\dot{L}\rangle \lambda+\sum_{k, l} L_{k, l} e^{i k \chi_{r}+i l \chi_{\theta},} \\
\tilde{C}=C_{0}+\langle\dot{C}\rangle \lambda+\sum_{k, l} C_{k, l} e^{i k \chi_{r}+i l \chi_{\theta}},
\end{gathered}
$$

where $\mathcal{E}_{0}^{a}$ denote the initial values at $\lambda=0 .{ }^{1}\left\langle\dot{\mathcal{E}}^{a}\right\rangle$ and $\mathcal{E}_{k, l}^{a}$ are of order $(\mu / M)^{2}$ and due to the self-force.

The orbital evolution can be derived. from (2.1), (2.2), (3.4), and (3.5). Following our notation convention introduced above, the inspiralling world line is denoted by $\tilde{x}^{\alpha}$. We define the $r$ - and $\theta$-motions by

$$
\tilde{r}=\sum_{k} \tilde{r}_{k} e^{i k \tilde{\chi}_{r}}, \quad \tilde{\theta}=\sum_{l} \tilde{\theta}_{l} e^{i l \tilde{\chi}_{\theta}},
$$

where the expansion coefficients $\tilde{r}_{k}$ and $\tilde{\theta}_{l}$ are the same as those in (2.3) and (2.4), but they are functions of $\tilde{\mathcal{E}}^{a}$ instead of $\mathcal{E}^{a}$. The evolution equations for $\tilde{\chi}_{r}$ and $\tilde{\chi}_{\theta}$ are given by $\frac{d}{d \lambda} \tilde{\chi}_{r}=\tilde{\Upsilon}_{r}-\frac{\sum_{k}\left(d_{\lambda} \tilde{r}_{k}\right) e^{i k \tilde{\chi}_{r}}}{\sum_{k^{\prime}} i k^{\prime} \tilde{r}_{k^{\prime}} e^{i k^{\prime} \tilde{\chi}_{r}}}, \quad \frac{d}{d \lambda} \tilde{\chi}_{\theta}=\tilde{\Upsilon}_{\theta}-\frac{\sum_{l}\left(d_{\lambda} \tilde{\theta}_{l}\right) e^{i l \tilde{\chi}_{\theta}}}{\sum_{l^{\prime}} i l^{\prime} \tilde{\theta}_{l^{\prime}} e^{i l^{\prime} \tilde{\chi}_{\theta}}}$,

where $d_{\lambda}=(d / d \lambda)$ acts on $\tilde{\mathcal{E}}^{a}$ of $\tilde{r}_{k}$ and $\tilde{\theta}_{l}$. The effective

\footnotetext{
${ }^{1}$ We choose $\mathcal{E}_{0,0}^{a}$, such that $\sum_{k, l} \mathcal{E}_{k, l}^{a} e^{i k \chi_{r}+i l \chi_{\theta}}=0$ when $\lambda=0$.
}

frequencies $\tilde{Y}_{r}$ and $\tilde{Y}_{\theta}$ are the same as $Y_{r}$ and $\Upsilon_{\theta}$ but are functions of $\tilde{\mathcal{E}}^{a}$ instead of $\mathcal{E}^{a}$. Similarly, we define the $t$ and $\phi$-motions by

$$
\begin{aligned}
& \tilde{t}=\tilde{\chi}_{t}+\sum_{k} \tilde{t}_{k}^{r} e^{i k \tilde{\chi}_{r}}+\sum_{l} \tilde{t}_{l}^{\theta} e^{i l \chi_{\theta}}, \\
& \tilde{\phi}=\tilde{\chi}_{\phi}+\sum_{k} \tilde{\phi}_{k}^{r} e^{i k \chi_{r}}+\sum_{l} \tilde{\phi}_{l}^{\theta} e^{i l \chi_{\theta}} .
\end{aligned}
$$

Then, the evolution equations for $\tilde{\chi}_{t}$ and $\tilde{\chi}_{\phi}$ are

$$
\begin{aligned}
\frac{d}{d \lambda} \tilde{\chi}_{t}= & \tilde{\Upsilon}_{t}+\sum_{k}\left\{i k\left(d_{\lambda} \tilde{\chi}_{r}-\tilde{\Upsilon}_{r}\right) \tilde{t}_{k}^{r}-d_{\lambda} \tilde{t}_{k}^{r}\right\} e^{i k \tilde{\chi}_{r}} \\
& +\sum_{l}\left\{i l\left(d_{\lambda} \tilde{\chi}_{\theta}-\tilde{\Upsilon}_{\theta}\right) \tilde{t}_{l}^{\theta}-d_{\lambda} \tilde{t}_{l}^{\theta}\right\} e^{i l \tilde{\chi}_{\theta}}, \\
\frac{d}{d \lambda} \tilde{\chi}_{\phi}= & \tilde{\Upsilon}_{\phi}+\sum_{k}\left\{i k\left(d_{\lambda} \tilde{\chi}_{r}-\tilde{\Upsilon}_{r}\right) \tilde{\phi}_{k}^{r}-d_{\lambda} \tilde{\phi}_{k}^{r}\right\} e^{i k \tilde{\chi}_{r}} \\
& +\sum_{l}\left\{i l\left(d_{\lambda} \tilde{\chi}_{\theta}-\tilde{\Upsilon}_{\theta}\right) \tilde{\phi}_{l}^{\theta}-d_{\lambda} \tilde{\phi}_{l}^{\theta}\right\} e^{i l \tilde{\chi}_{\theta}},
\end{aligned}
$$

where the effective frequencies $\tilde{Y}_{t}$ and $\tilde{Y}_{\phi}$ are the same as $Y_{t}$ and $Y_{\phi}$ but are functions of $\tilde{\mathcal{E}}^{a}$ instead of $\mathcal{E}^{a}$.

We now derive the formal expression of $\tilde{\chi}_{\alpha}$ by perturbation. Recall that $\tilde{\chi}_{\alpha}-\chi_{\alpha}$ is of order $\mu / M$ by definition. The formal expressions (3.7), (3.9), and (3.10) can therefore be written in the form

$$
\frac{d}{d \lambda} \tilde{\chi}_{\alpha}=\Upsilon_{\alpha(0)}+\left\langle\dot{\Upsilon}_{\alpha}\right\rangle \lambda+\sum_{k, l} \Upsilon_{\alpha(k, l)} e^{i k \chi_{r}+i l \chi_{\theta}}
$$

where $\Upsilon_{\alpha(0)}=\left.\Upsilon_{\alpha}\right|_{\mathcal{E}=\mathcal{E}_{0}}$ is for the background orbital evolution. The formal expression of $\tilde{\chi}_{\alpha}$ becomes

$$
\begin{aligned}
\tilde{\chi}_{\alpha}= & \Upsilon_{\alpha(0)}\left(\lambda-\lambda^{\alpha}\right)+\frac{1}{2}\left\langle\dot{\Upsilon}_{\alpha}\right\rangle \lambda^{2}+\Upsilon_{\alpha(0,0)} \lambda \\
& +\sum_{k, l} \tilde{\chi}_{\alpha(k, l)} e^{i k \chi_{r}+i l \chi_{\theta}},
\end{aligned}
$$

where we set such that $\tilde{\chi}_{\alpha}=\chi_{\alpha}$ when $\lambda=0 .^{2}$ This expression has two key features. One is the linear growth from $\frac{1}{2}\left\langle\dot{\Upsilon}_{\alpha}\right\rangle \lambda^{2}$. This effect comes from the linear growth of $\mathcal{E}^{a}$ since $\left\langle\dot{\Upsilon}_{\alpha}\right\rangle=\left(\partial Y_{\alpha} / \partial \mathcal{E}^{a}\right)\left\langle\dot{\mathcal{E}}^{a}\right\rangle$. This is expected to give the dominant phase evolution of the gravitational waveform because of the quadratic growth of the phase. It is the reason that the orbit's phase deviates from that of a geodesic on the dephasing time scale $(\propto 1 / \sqrt{\mu})$, while its frequencies deviate on the radiation reaction time scale $(\propto$ $1 / \mu)$. The second feature is a small shift of the time averaged frequencies by $\Upsilon_{\alpha(0,0)}$. The phase evolution due to this effect will accumulate in time [7], however it remains small over the radiation reaction time and is therefore not likely to be observable [6].

\footnotetext{
${ }^{2}$ This is achieved by choosing $\tilde{\chi}_{\alpha(0,0)}$ such that $\sum_{k, l} \tilde{\chi}_{\alpha(k, l)} e^{i k \chi_{r}+i l \chi_{\theta}}=0$ at $\lambda=0$.
} 


\section{THE SECOND ORDER METRIC PERTURBATION}

In this section we derive formal solutions of the second order Einstein equation (1.5). By using the same gauge condition as we solve (1.4), we can formally integrate (1.5) with the tensor Green's function (2.7). We separate the second order metric perturbation into pieces

$$
g_{\alpha \beta}^{[2]}=g_{\alpha \beta}^{[2] \mathrm{NL}}+g_{\alpha \beta}^{[2] \mathrm{SF}},
$$

where $g_{\alpha \beta}^{[2] \mathrm{NL}}$ is due to the nonlinearity of the Einstein equation and $g_{\alpha \beta}^{[2] \mathrm{SF}}$ is from the perturbation of the source. (1.5) then becomes

$$
\begin{aligned}
& G^{[1] \mu \nu}\left[\mathbf{g}^{(2) \mathrm{NL}}\right]=-G^{[2] \mu \nu}\left[\mathbf{g}^{(1)}, \mathbf{g}^{(1)}\right], \\
& G^{[1] \mu \nu}\left[\mathbf{g}^{(2) \mathrm{SF}}\right]=8 \pi T^{[2] \mu \nu} .
\end{aligned}
$$

We first discuss the formal calculation of $\mathbf{g}^{[2] \mathrm{NL}}$. By the Green's method, we have

$g_{\alpha \beta}^{[2] \mathrm{NL}}(x)=-\int_{-\infty<r^{*}<\infty} d x^{\prime 4} G_{\alpha \beta \mu \nu} G^{[2] \mu \nu}\left[\mathbf{g}^{(1)}, \mathbf{g}^{(1)}\right]$,

where the domain of radial integration is outside the outer horizon and inside the future infinity; with $r^{*}=\int d r\left(r^{2}+\right.$ $\left.a^{2}\right) /\left(r^{2}-2 M r+a^{2}\right)$, this is defined as $-\infty<r^{*}<\infty$. Because the Kerr geometry is stationary an axisymmetric, the differential operator $G^{[2] \mu \nu}[\mathbf{h}, \mathbf{k}]$ does not include terms that explicitly depend on $t$ and $\phi$. Therefore, the formal expression of the source term is

$$
\begin{aligned}
G^{[2] \mu \nu}\left[\mathbf{g}^{(1)}, \mathbf{g}^{(1)}\right]= & \sum_{k, l, m} e^{-i \omega_{(k, l, m)} t+i m \phi} G_{(k, l, m)}^{[2] \mu \nu}(r, \theta) \\
& \times e^{i\left(k \omega_{r} t^{r}+l \omega_{\theta} t^{\theta}+m \omega_{\phi} t^{\phi}\right)},
\end{aligned}
$$

where we have used (2.10). Using the Green's function of the form (2.7), one may obtain the formal expression

$$
\begin{aligned}
g_{\alpha \beta}^{[2] \mathrm{NL}}(x)= & \sum_{k, l, m} e^{-i \omega_{(k, l, m)} t+i m \phi} g_{(k, l, m)}^{[2] \alpha \beta}(r, \theta) \\
& \times e^{i\left(k \omega_{r} r^{r}+l \omega_{\theta} t^{\theta}+m \omega_{\phi} t^{\phi}\right)} .
\end{aligned}
$$

We see that $\mathbf{g}^{[2] \mathrm{NL}}$ has the same formal expression as the first order term (2.10). This part of the second order metric perturbation changes on the dynamical time scale of the orbit which is much shorter than the radiation reaction time scale. This means that $\mathbf{g}^{[2] \mathrm{NL}}$ does not include radiation reaction effects, and that $\mathbf{g}^{[2] \mathrm{NL}}$ describes the nonlinear effect of the Einstein equation. This effect makes a small correction to the wave amplitude of the linear metric perturbation, (2.10), and is not likely to be observable.

Although this conclusion may look reasonable, this argument is not yet well supported mathematically because (4.3) is actually divergent. In order to evaluate the formal expression of $\mathbf{g}^{[2] \mathrm{NL}}$, it is necessary to see the possible change due to the regularization calculation. The integration of (4.3) has two kinds of divergences. One was pointed out in Ref. [8], where it was referred to as an ultraviolet divergence. Near the particle, the singular behavior of the linear metric perturbation under the harmonic gauge condition is $\mathbf{g}^{[1]} \propto \mu / R$, where $R$ is the spatial distance from the particle in the local inertia frame. Because the Einstein tensor is a second order differential operator, we have $G^{[2] \mu \nu}\left[\mathbf{g}^{(1)}, \mathbf{g}^{(1)}\right] \propto \mu^{2} / R^{4}$. The Green's function behaves as $\propto 1 / R$. Therefore the spatial integral in (4.3) diverges as $1 / R^{2}$ near the orbit. The other kind of divergence, an infrared divergence in the field theory terminology, is due to the fact that the graviton is a massless particle. Because the geodesic source of the linear metric perturbation is a stable orbit, it can radiate an infinite amount of energy from the infinite past, which is stored in a Cauchy surface. This becomes a source for the second order perturbation given by (4.3). At spatial infinity, the linear metric perturbation of a specific frequency under the harmonic gauge condition behaves asymptotically as $\mathbf{g}^{[1]} \sim \mu e^{-i \omega(t-r)} / r$ where $r$ is the radius in asymptotically flat coordinates. This means that $G^{[2] \mu \nu}\left[\mathbf{g}^{(1)}, \mathbf{g}^{(1)}\right] \sim \mu^{2} \omega \omega^{\prime} e^{-i\left(\omega+\omega^{\prime}\right)(t-r)} / r^{2}$, and the spatial integral of (4.3) diverges as $\ln (r)$ at infinity. In Appendix $\mathrm{B}$ and $\mathrm{C}$, we introduce the regularization prescription for (4.3) and show that it does not alter the result. By applying a gauge transformation, the result should apply for metric perturbations under broader gauge conditions.

We next discuss the formal calculation of $\mathbf{g}^{[2] \mathrm{SF}}$. In order to identify the stress-energy tensor for the second order metric perturbation, we recall the derivation of the selfforce by the linear metric perturbation. In Ref. [4], the selfforce was derived using a matched asymptotic expansion of the black hole metric for the particle and the linear metric perturbation away from the orbit. The singular part of the linear metric perturbation around the particle (that is, the part of $\mathbf{g}$ that diverges as $\mu / R$ ) is correctly matched to the corresponding singular behavior of the black hole metric. In order to match the regular part of the linear metric perturbation with the corresponding part of the black hole metric, it is necessary to account for the motion of the black hole with respect to the local inertia frame of the background metric. The result is the regular equation of motion. This suggests that the stress-energy tensor to the second order perturbation can be written as

$$
T^{[1] \mu \nu}+T^{[2] \mu \nu}=\mu \int d \tau \tilde{v}^{\mu} \tilde{\boldsymbol{v}}^{\nu} \frac{\delta(x-\tilde{x}(\tau))}{\sqrt{-\left|g^{[0]}\right|}},
$$

where $\tilde{x}^{\alpha}$ and $\tilde{v}^{\alpha}$ are the position and 4-velocity of the orbit derived in Sec. III.

The metric perturbation is then

$$
g_{\alpha \beta}^{[1]}+g_{\alpha \beta}^{[2] \mathrm{SF}}=8 \pi \mu \int d \lambda\left(\frac{d \tau}{d \lambda}\right) G_{\alpha \beta, \mu \nu}(x, \tilde{x}(\lambda)) \tilde{v}^{\mu} \tilde{\boldsymbol{v}}^{\nu} .
$$

Recall our definitions (3.6) and (3.8) for the orbital coor- 
dinates. Here, we use the same formal expressions (2.3), (2.4), (2.5), and (2.6) for the geodesic, but we replace $\mathcal{E}^{a}$ and $\chi_{\alpha}$ by $\tilde{\mathcal{E}}^{a}$ and $\tilde{\chi}_{\alpha}$. Thus we can rewrite (4.7) by replacing $\mathcal{E}^{a}$ and $\chi_{\alpha}$ of (A3) with $\tilde{\mathcal{E}}^{a}$ and $\tilde{\chi}_{\alpha}$

$$
\begin{aligned}
g_{\alpha \beta}^{[1]}+g_{\alpha \beta}^{[2] \mathrm{SF}}= & 8 \pi \mu \int d \lambda \sum_{\omega, k, l, m} e^{-i \omega t+i m \phi} \tilde{h}_{\alpha \beta}^{\omega, k, l, m}(r, \theta ; \tilde{\mathcal{E}}) \\
& \times e^{i \omega \tilde{\chi}_{t}-i m \tilde{\chi}_{\phi}-i k \tilde{\chi}_{r}-i l \tilde{\chi}_{\theta}}
\end{aligned}
$$

where $\tilde{h}_{\alpha \beta}^{\omega, k, l, m}$ is defined in the same way as $h_{\alpha \beta}^{\omega, k, l, m}$, but with $\mathcal{E}^{a}$ replaced by $\tilde{\mathcal{E}}^{a}$.

We now discuss the effect of the self-force on (4.8) by perturbation. There are two kinds of effects. One is from the dependence of the expansion coefficients $\tilde{h}_{\alpha \beta}^{\omega, k, l, m}$ of $\tilde{\mathcal{E}}^{a}$, and the other is from the phase function $\tilde{\chi}_{\alpha}$ in the exponentials.

We first look at the effect of the expansion coefficients $\tilde{h}_{\alpha \beta}^{\omega, k, l, m}$. Using (3.4) and (3.5), the expansion coefficients become

$$
\begin{aligned}
\tilde{h}_{\alpha \beta}^{\omega, k, l, m}(r, \theta ; \tilde{\mathcal{E}}) \rightarrow & \tilde{h}_{\alpha \beta}^{\omega, k, l, m}\left(r, \theta ; \mathcal{E}_{0}\right) \\
& +\frac{d}{d \tilde{\mathcal{E}}^{a}} \tilde{h}_{\alpha \beta}^{\omega, k, l, m}\left(r, \theta ; \mathcal{E}_{0}\right)\left\langle\dot{\mathcal{E}}^{a}\right\rangle \lambda,
\end{aligned}
$$

where we have ignored the oscillating part of $\tilde{\mathcal{E}}^{a}$. (The metric perturbation due to the oscillating part of $\tilde{\mathcal{E}}^{a}$ can be written in the form (2.10), and does not show any new characteristic feature.) One can see that the expansion coefficients grow linearly with $\lambda$. This is because of the linear growth of $\mathcal{E}^{a}$ caused by the self-force. This leads to the linearly growing/decaying feature of the wave amplitude through the $\mathcal{E}^{a}$-dependence of $h_{\alpha \beta}^{\omega_{(k, l, m)}, k, l, m}$ as $^{3}$

$$
\begin{gathered}
\frac{2 \pi}{\Upsilon_{t(0)}} 8 \pi \mu \sum_{k, l, m} e^{-i \omega_{(k, l, m)} t+i m \phi}\left(h_{\alpha \beta}^{\left.\omega_{(k, l, m}\right), k, l, m}(r, \theta)\right. \\
\left.\quad+\dot{h}_{\alpha \beta}^{\omega_{(k, l, m)}, k, l, m}(r, \theta) t\right) e^{i\left(k \omega_{r} r^{r}+l \omega_{\theta} t^{\theta} m \omega_{\phi} t^{\phi}\right)},
\end{gathered}
$$

where $\dot{h}_{\alpha \beta}^{\omega_{(k, l, m)}, k, l, m}=\left(d \tilde{h}_{\alpha \beta}^{\omega, k, l, m} / d \mathcal{E}^{a}\right)\left\langle\dot{\mathcal{E}}^{a}\right\rangle / \Upsilon_{t}$. This formal expression for the waveform has a new feature, that is, the wave amplitude changes on the radiation reaction time scale.

${ }^{3}$ The actual procedure of integration is

$$
\begin{aligned}
\int d \omega e^{-i \omega t} & \int d t^{\prime} t^{\prime} e^{i\left(\omega-\omega_{0}\right) t^{\prime}} \\
& =\int d \omega e^{-i \omega t}(-i) \frac{d}{d \omega} \int d t^{\prime} e^{i\left(\omega-\omega_{0}\right) t^{\prime}} \\
& =(2 \pi) \int d \omega e^{-i \omega t}(-i) \frac{d}{d \omega} \delta\left(\omega-\omega_{0}\right) \\
& =(2 \pi) \int d \omega \delta\left(\omega-\omega_{0}\right) i \frac{d}{d \omega} e^{-i \omega t} \\
& =(2 \pi) t e^{-i \omega_{0} t} .
\end{aligned}
$$

We next see the effect of $\tilde{\chi}_{\alpha}$ in the exponential of (4.8). Using (3.12) this exponential has a quadratically growing feature

$$
\begin{aligned}
e^{i \omega \tilde{\chi}_{t}-i m \tilde{\chi}_{\phi}-i k \tilde{\chi}_{r}-i l \tilde{\chi}_{\theta} \rightarrow} & e^{i \omega \tilde{\Upsilon}_{t(0)} \lambda-i m \tilde{\Upsilon}_{\phi(0)} \lambda-i k \tilde{\Upsilon}_{r(0)} \lambda-i l \tilde{Y}_{\theta(0)} \lambda} \\
& \times e^{-i \omega \Upsilon_{t(0)} \lambda^{t}+i m \Upsilon_{\phi(0)} \lambda^{\phi}+i k Y_{r(0)} \lambda^{r}+i l \Upsilon_{\theta(0)} \lambda^{\theta}} \\
& \times\left\{1+\left(i \omega\left\langle\dot{\Upsilon}_{t}\right\rangle-i m\left\langle\dot{\Upsilon}_{\phi}\right\rangle\right.\right. \\
& \left.\left.-i k\left\langle\dot{\Upsilon}_{r}\right\rangle-i l\left\langle\dot{\Upsilon}_{\theta}\right\rangle\right) \frac{\lambda^{2}}{2}\right\}
\end{aligned}
$$

where we have ignored the oscillating part of $\tilde{\chi}_{\alpha}$ (Again, the metric perturbation due to the oscillating part of $\tilde{\chi}_{\alpha}$ can be written in the form (2.10), and does not show any new characteristic feature.) and we use the renormalized frequencies $\tilde{\Upsilon}_{\alpha(0)}=\Upsilon_{\alpha(0)}+\Upsilon_{\alpha(0,0)}$. We obtain the formal expression as ${ }^{4}$

$$
\begin{aligned}
\frac{2 \pi}{\Upsilon_{t(0)}} 8 \pi & \mu \sum_{k, l, m} e^{-i\left(\tilde{\omega}_{(k, l, m)} t+\dot{\omega}_{(k, l, m)} t^{2} / 2\right)+i m \phi} h_{\alpha \beta}^{\tilde{\omega}_{(k, l, m)}, k, l, m}(r, \theta) \\
& \times e^{i\left(k \omega_{r} t^{r}+l \omega_{\theta} t^{\theta} m \omega_{\phi} t^{\phi}\right)},
\end{aligned}
$$

where we used

$$
\begin{gathered}
\tilde{\omega}_{(k, l, m)}=m \frac{\tilde{\Upsilon}_{\phi(0)}}{\tilde{\Upsilon}_{t(0)}}+k \frac{\tilde{\Upsilon}_{r(0)}}{\tilde{\Upsilon}_{t(0)}}+l \frac{\tilde{\Upsilon}_{\theta(0)}}{\tilde{\Upsilon}_{t(0)}}, \\
\dot{\omega}_{(k, l, m)}=m\left(\frac{\left\langle\dot{\Upsilon}_{\phi}\right\rangle}{\Upsilon_{t(0)}^{2}}-\frac{\Upsilon_{\phi(0)}\left\langle\dot{\Upsilon}_{t}\right\rangle}{\Upsilon_{t(0)}^{3}}\right)+k\left(\frac{\left\langle\dot{\Upsilon}_{r}\right\rangle}{\Upsilon_{t(0)}^{2}}-\frac{\Upsilon_{r(0)}\left\langle\dot{\Upsilon}_{t}\right\rangle}{\Upsilon_{t(0)}^{3}}\right) \\
+l\left(\frac{\left\langle\dot{\Upsilon}_{\theta}\right\rangle}{\Upsilon_{t(0)}^{2}}-\frac{\Upsilon_{\theta(0)}\left\langle\dot{\Upsilon}_{t}\right\rangle}{\Upsilon_{t(0)}^{3}}\right) .
\end{gathered}
$$

From the time-dependence of the exponent, one can see two key features of the wave phase. First, the quadratic growth of the wave phase on the dephasing time due to the linear growth of the fundamental frequencies. Second, the linear growth of the wave phase over the radiation reaction time due to the renormalization of the fundamental frequencies. Both features have been seen from the orbital evolution due to the self-force as we argued in Sec. III.

$$
\begin{aligned}
& { }^{4} \text { The actual procedure of integration is } \\
& \begin{aligned}
\int d \omega e^{-i \omega t} & \int d t^{\prime} t^{\prime 2} e^{i\left(\omega-\omega_{0}\right) t^{\prime}} \\
& =\int d \omega e^{-i \omega t}(-1) \frac{d^{2}}{d \omega^{2}} \int d t^{\prime} e^{i\left(\omega-\omega_{0}\right) t^{\prime}} \\
& =(2 \pi) \int d \omega e^{-i \omega t}(-1) \frac{d^{2}}{d \omega^{2}} \delta\left(\omega-\omega_{0}\right) \\
& =(2 \pi) \int d \omega \delta\left(\omega-\omega_{0}\right)(-1) \frac{d^{2}}{d \omega^{2}} e^{-i \omega t} \\
& =(2 \pi) t^{2} e^{-i \omega_{0} t} .
\end{aligned}
\end{aligned}
$$


The results of (4.11) and (4.14) suggest that the gravitational field might be well approximated by the form

$$
\mu \sum_{k, l, m} h_{\alpha \beta}^{k, l, m}(r, \theta ; \tilde{\mathcal{E}}(t)) e^{-i k \tilde{\chi}_{r}(t)-i l \tilde{\chi}_{\theta}(t)-i m \tilde{\chi}_{\phi}(t)},
$$

where $\tilde{\mathcal{E}}$ and $\tilde{\chi}_{i}(i=r, \theta, \phi)$ are functions of the BoyerLindquist coordinate time $t$ and the radiation reaction effect is included in the waveform through those functions.

\section{CONCLUSION}

In this paper, we discuss the modulation of the gravitational waveform due to gravitational radiation reaction. Since the linear metric perturbation is induced only by the background geodesic, it is necessary to calculate the second order metric perturbation in order to see the radiation reaction effect. A more rigorous calculation of the second order metric perturbation is not yet available. For this reason, we have considered only a qualitative study, extending the technique of formal calculation used to obtain the linear metric perturbation in Ref. [1]. The advantage of this technique is that one can grasp some key features of the gravitational waveform without a complicated calculation.

There are two kinds of source terms for the second order metric perturbation. One is the nonlinear term of the linear metric perturbation $G^{[2] \mu \nu}\left[\mathbf{g}^{(1)}, \mathbf{g}^{(1)}\right]$. Because the formal calculation of this term is triperiodic, the part of the second order metric perturbation (4.5) is also triperiodic. The waveform induced by this part has exactly the same spectral form as that of the linear metric perturbation. This part simply changes the amplitude of the waves, therefore, describing the correction to the wave propagation due to the nonlinearity of the Einstein equation. The other kind of source term is the point source due to the orbital deviation from the background geodesic, $T^{[2] \mu \nu}$. The part of the second order metric perturbation induced by this source has two new features; the linear growth of the wave amplitude over the radiation reaction time scale [See (4.11).] and the quadratic growth of the wave phase over the dephasing time scale [See (4.14).]. From the derivations of (4.11) and (4.14), it should be clear that these features reflect the secular effect of the orbital evolution due to the self-force discussed in Sec. III.

These results suggest that the effect of radiation reaction on the waveform are included in templates of the form (4.17), which is the linear metric perturbation with the geodesic constants replaced by those which evolve adiabatically due to the self-force. This result agrees with the procedure of the adiabatic approximation. However, strictly speaking the perturbation scheme is valid over a dephasing time [6], and over that time scale, only the feature of the quadratic growth of the wave phase can be identified as a secular effect. Beyond this time scale, it is necessary to account for the secular effect of the self-force due to the second order metric perturbation. One can only discuss those linearly growing features in a gauge invariant way after calculating the third order metric perturbation.

Because the perturbation scheme we use is limited to the dephasing time scale, it is not an appropriate method for calculating gravitational waveforms. In order to solve this problem, we propose an adiabatic expansion that is a systematic perturbation of a field theory coupled to a particle [6]. Because this expansion recovers the adiabatic approximation at leading order, our result here supports the validity of this new expansion method. The application of this technique to the adiabatic expansion shall be discussed elsewhere.

\section{ACKNOWLEDGMENTS}

We thank Professor Richard Price for fruitful discussion. We thank Dr. Steve Drasco for carefully reading the material. This work is supported by NSF grant PHY-0601459, NASA grant NNX07AH06G, NNG04GK98G and the Brinson Foundation.

\section{APPENDIX A: FORMAL EXPRESSION OF THE LINEAR METRIC PERTURBATION}

In this appendix, we review the formal calculation of the linear metric perturbation given as (2.9). With the ansatz (2.7), we decompose the Green's function as

$$
G_{\alpha \beta \mu \nu}\left(x, x^{\prime}\right)=\sum_{\omega, m} g_{\alpha \beta \mu \nu}^{\omega, m}\left(r, r^{\prime} ; \theta, \theta^{\prime}\right) e^{-i \omega\left(t-t^{\prime}\right)+i m\left(\phi-\phi^{\prime}\right)} .
$$

We plug this into (2.9) and we have

$$
\begin{aligned}
g_{\alpha \beta}^{[1]}(x)= & 8 \pi \mu \int d \lambda\left(\frac{d \tau}{d \lambda}\right) \sum_{\omega, m} g_{\alpha \beta \mu \nu}^{\omega, m}(r, \bar{r} ; \theta, \bar{\theta}) v^{\mu} v^{\nu} \\
& \times e^{-i \omega(t-\bar{t})+i m(\phi-\bar{\phi})} .
\end{aligned}
$$

At this point, we recall the periodic feature of the orbit (2.3), (2.4), (2.5), and (2.6). It is easy to see that $d \tau / d \lambda$ and $g_{\alpha \beta \mu \nu}^{\omega, m}(r, \bar{r} ; \theta, \bar{\theta})$ can be expanded as a discrete Fourier series in $e^{-i k \chi_{r}-i l \chi_{\theta}}$. From (2.1) and (2.2), $v^{\mu} v^{\nu}$ can be similarly expanded. As for $e^{i \omega \bar{t}-i m \bar{\phi}}$, one may expand by the same discrete Fourier series except the factor $e^{i \omega \chi_{t}-i m \chi_{\phi}}$. In summary, one can formally rewrite (A2) as

$$
\begin{aligned}
g_{\alpha \beta}^{[1]}(x)= & 8 \pi \mu \int d \lambda \sum_{\omega, k, l, m} e^{-i \omega t+i m \phi} h_{\alpha \beta}^{\omega, k, l, m}(r, \theta) \\
& \times e^{i \omega \chi_{t}-i m \chi_{\phi}-i k \chi_{r}-i l \chi_{\theta} .} .
\end{aligned}
$$

By integrating over $\lambda$, we obtain

$$
\begin{aligned}
g_{\alpha \beta}^{[1]}(x)= & \frac{2 \pi}{\Upsilon_{t}} 8 \pi \mu \sum_{k, l, m} e^{-i \omega_{(k, l, m)} t+i m \phi} h_{\alpha \beta}^{\left.\omega_{(k, l, m)}\right), l, l, m}(r, \theta) \\
& \times e^{i m \Upsilon_{\phi}\left(\lambda^{\phi}-\lambda^{t}\right)+i k \Upsilon_{r}\left(\lambda^{r}-\lambda^{t}\right)+i l \Upsilon_{\theta}\left(\lambda^{\theta}-\lambda^{t}\right)},
\end{aligned}
$$

which is equal to (2.10). 


\section{APPENDIX B: REGULARIZATION OF THE ULTRAVIOLET DIVERGENCE}

We use the regularization prescription for the ultraviolet divergence proposed in Ref. [8]. By the consistency of the matched asymptotic expansion, we know that the divergence behavior of the second order metric perturbation becomes $\mathbf{g}^{[2] \mathrm{NL}} \propto \mu^{2} / R^{2}$, with respect to the local inertial coordinates in the neighborhood of the geodesic. ( $R$ is the spatial distance from the particle in the local inertia frame.) The idea in Ref. [8] is to subtract this divergence by using the quadratic combination of the scalar field induced by a point particle and calculate the remaining regular part by the Green's method. We use the scalar field that satisfies

$$
\square \Phi=\int d \tau \frac{\delta(x-\bar{x}(\tau))}{\sqrt{-\left|g^{[0]}\right|}},
$$

under the retarded boundary condition, where $\bar{x}^{\mu}(\tau)$ is the same geodesic used in (2.8) for the linear metric perturbation. The resulting scalar field has the divergence behavior $\Phi \propto 1 / R$ near the geodesic. With appropriate tensors $k_{\alpha \beta}^{[2]}$ and $k_{\alpha \beta}^{[1]}$, which are regular around the orbit, one may construct

$$
g_{\alpha \beta}^{(S)[2] \mathrm{NL}}=k_{\alpha \beta}^{[2]} \Phi^{2}+k_{\alpha \beta}^{[1]} \Phi,
$$

such that $\mathbf{g}^{[2] \mathrm{NL}}$ and $\mathbf{g}^{(S)[2] \mathrm{NL}}$ have the same singular behavior near the geodesic. We define the remaining part by

$$
g_{\alpha \beta}^{(R)[2] \mathrm{NL}}=g_{\alpha \beta}^{[2] \mathrm{NL}}-g_{\alpha \beta}^{(S)[2] \mathrm{NL}},
$$

which satisfies

$$
G^{[1] \mu \nu}\left[\mathbf{g}^{(R)(2) \mathrm{NL}}\right]=-G^{[1] \mu \nu}\left[\mathbf{g}^{(S)(2) N L}\right]-G^{[2] \mu \nu}\left[\mathbf{g}^{(1)}, \mathbf{g}^{(1)}\right] .
$$

Because $g_{\alpha \beta}^{(R)[2] \mathrm{NL}}$ is regular, the RHS of (B4) must be regular near the geodesic, and $\mathbf{g}^{(R)(2) \mathrm{NL}}$ can be written as

$$
\begin{aligned}
g_{\alpha \beta}^{(R)[2] \mathrm{NL}}(x)= & -\int_{-\infty<r^{*}<\infty} d^{4} x^{\prime} G_{\alpha \beta \mu \nu}\left(G^{[1] \mu \nu}\left[\mathbf{g}^{(S)(2) \mathrm{NL}}\right]\right. \\
& \left.+G^{[2] \mu \nu}\left[\mathbf{g}^{(1)}, \mathbf{g}^{(1)}\right]\right) .
\end{aligned}
$$

In order to see the formal expression for (B5), it is necessary to know the formal expression for (B2). The expression for $\Phi$ can be obtained in a similar manner as (2.10). Because the scalar Green's function of (B1) can be written in the form

$$
G\left(x, x^{\prime}\right)=G\left(t-t^{\prime}, \phi-\phi^{\prime} ; r, r^{\prime}, \theta, \theta^{\prime}\right),
$$

similarly to (2.7), the formal expression of $\Phi$ becomes

$$
\begin{aligned}
\Phi(x)= & \sum_{k, l, m} e^{-i \omega_{(k, l, m)} t+i m \phi} \Phi_{(k, l, m)}\left(r, \theta ; \mathcal{E}^{a}\right) \\
& \times e^{i\left(k \omega_{r} t^{r}+l \omega_{\theta} t^{\theta}+m \omega_{\phi} t^{\phi}\right)} .
\end{aligned}
$$

The structure of the singularity depends only on the local background geometry, and the components of $k_{\alpha \beta}^{[1]}$ and $k_{\alpha \beta}^{[2]}$ can be constructed only from the background curvature along the orbit. Therefore, we have

$$
\begin{aligned}
k_{\alpha \beta}^{[n]}= & \sum_{k, l, m} e^{-i \omega_{(k, l, m)} t+i m \phi} k_{\alpha \beta(k, l, m)}^{[n]}\left(r, \theta ; \mathcal{E}^{a}\right) \\
& \times e^{i\left(k \omega_{r} t^{r}+l \omega_{\theta} t^{\theta}+m \omega_{\phi} t^{\phi}\right)},
\end{aligned}
$$

for $n=1$, 2. In summary, the RHS of (B4) has the same formal structure as (4.4).

\section{APPENDIX C: REGULARIZATION OF THE INFRARED DIVERGENCE}

Though we can remove the ultraviolet divergence by calculating (B5) instead of calculating (4.3), it does not guarantee that we can also remove the infrared divergence, i.e. the integration over the spatial volume of (B5) becomes divergent at the large spatial radius and at the black hole horizon.

A formal method of avoiding this divergence is to introduce the radial regulator $\Gamma$ to (B5) as

$$
\begin{aligned}
g_{\alpha \beta}^{(R)[2] \mathrm{NL}}(x ; \Gamma)= & -\int_{-\Gamma<r^{*}<\Gamma} d x^{\prime 4} G_{\alpha \beta \mu \nu}\left(G^{[1] \mu \nu}\left[\mathbf{g}^{(S)(2) \mathrm{NL}}\right]\right. \\
& \left.+G^{[2] \mu \nu}\left[\mathbf{g}^{(1)}, \mathbf{g}^{(1)}\right]\right) .
\end{aligned}
$$

By definition, $\mathbf{g}^{(R)[2] \mathrm{NL}}(x ; \Gamma)$ satisfies (B4) for $-\Gamma<r^{*}<$ $\Gamma$, and it diverges as $\mathbf{g}^{(R)[2] \mathrm{NL}}(x ; \Gamma) \propto \ln (\Gamma)$, when $\Gamma \rightarrow \infty$.

The counter term to cancel this divergence $\mathbf{g}^{(C)[2] \mathrm{NL}}(x ; \Gamma)$ must satisfy the vacuum linearized Einstein equation so that $\mathbf{g}^{(R)[2] \mathrm{NL}}+\mathbf{g}^{(C)[2] \mathrm{NL}}$ still satisfies (B4). Because $\mathbf{g}^{[2] \mathrm{NL}}$ is intrinsically divergent along the orbit, one may consider a point source moving along the geodesic which induces $\mathbf{g}^{(C)[2] \mathrm{NL}}(x ; \Gamma)$ as

$$
T^{[2] \mathrm{NL} \mu \nu}(x ; \Gamma)=\ln (\Gamma) \mu^{2} \int d \tau^{[2] \mu \nu} \frac{\delta(x-\bar{x}(\tau))}{\sqrt{-\left|g^{[1]}\right|}},
$$

where $\bar{x}^{\alpha}(\tau)$ is the same geodesic as in (2.8). We have the counterterm

$$
\begin{aligned}
g_{\alpha \beta}^{(C)[2] \mathrm{NL}}(x ; \Gamma) & =8 \pi \int d x^{\prime 4} G_{\alpha \beta \mu \nu}\left(x, x^{\prime}\right) T^{[2] \mathrm{NL} \mu \nu}(x ; \Gamma) \\
& =8 \pi \mu^{2} \ln (\Gamma) \int d \tau \hat{t}^{[2] \mu \nu} G_{\alpha \beta \mu \nu}(x, \bar{x}) .
\end{aligned}
$$

Here $\hat{t}^{[2] \mu \nu}$ is the tensor derivative operator defined along the orbit such that $\left(\mathbf{g}^{(R)[2] \mathrm{NL}}+\mathbf{g}^{(C)[2] \mathrm{NL}}\right)(x ; \Gamma)$ becomes finite everywhere except along the geodesic when $\Gamma \rightarrow \infty$.

We discuss the formal expression of $\lim _{\Gamma \rightarrow \infty}\left(\mathbf{g}^{(R)[2] \mathrm{NL}}+\right.$ $\left.\mathbf{g}^{(C)[2] \mathrm{NL}}\right)(x ; \Gamma)$ following this regularization prescription. For a finite $\Gamma$, as we may see from $(\mathrm{C} 1), \mathbf{g}^{(R)[2] \mathrm{NL}}(x ; \Gamma)$ has the same formal expression as (4.5) because the source term of (4.5) has the same formal expression as (4.4). By 
$\mathbf{g}^{(C)[2] \mathrm{NL}}(x ; \Gamma)$, we simplify subtract the linear divergent piece of $\mathbf{g}^{(R)[2] \mathrm{NL}}(x ; \Gamma)$, i.e. we have

$$
\mathbf{g}^{(C)[2] \mathrm{NL}}(x ; \Gamma)=-\ln (\Gamma) \lim _{\Gamma^{\prime} \rightarrow \infty} \frac{\mathbf{g}^{(R)[2] \mathrm{NL}}\left(x ; \Gamma^{\prime}\right)}{\ln \left(\Gamma^{\prime}\right)},
$$

and this operation does not alter the formal expression. Thus, by the infrared regularization, the part of the second order metric perturbation due to the nonlinear term of (1.5) has the formal expression of (4.5).
[1] Y. Mino, Phys. Rev. D 67, 084027 (2003).

[2] S. Drasco (unpublished).

[3] Recent development of the perturbative approach to the gravitational radation reaction is reviewed in Classical Quantum Gravity 22, S543 (2005).

[4] Y. Mino, M. Sasaki, and T. Tanaka, Phys. Rev. D 55, 3457 (1997); E. Poisson, Living Rev. Relativity 7, 6 (2004), http://www.livingreviews.org/lrr-2004-6; Classical Quantum Gravity 21, R153 (2004).

[5] L. Barack and A. Ori, Phys. Rev. D 61, 061502(R) (2000); L. Barack, Phys. Rev. D 62, 084027 (2000); 64, 084021 (2001); L. Barack, Y. Mino, H. Nakano, A. Ori, and M. Sasaki, Phys. Rev. Lett. 88, 091101 (2002); L. Barack and A. Ori, Phys. Rev. D 66, 084022 (2002); Y. Mino, H. Nakano, and M. Sasaki, Prog. Theor. Phys. 108, 1039 (2002); S. Detweiler and B. F. Whiting, Phys. Rev. D 67, 024025 (2003); L. Barack and A. Ori, Phys. Rev. D 67,
024029 (2003); Phys. Rev. Lett. 90, 111101 (2003); S. Detweiler, E. Messaritaki, and B. F. Whiting, Phys. Rev. D 67, 104016 (2003); D. H. Kim, arXiv:gr-qc/0402014; W. Hikida, H. Nakano, and M. Sasaki, Classical Quantum Gravity 22, S753 (2005); W. G. Anderson, E. E. Flanagan, and A. C. Ottewill, Phys. Rev. D 71, 024036 (2005); W. G. Anderson and A. G. Wiseman, Classical Quantum Gravity 22, S783 (2005); L. Barack and N. Sago, Phys. Rev. D 75, 064021 (2007); R. Haas, Phys. Rev. D 75, 124011 (2007).

[6] Y. Mino, Prog. Theor. Phys. 115, 43 (2006); 113, 733 (2005); Y. Mino and R. Price (unpublished).

[7] A. Pound and E. Poisson, arXiv:gr-qc/0708.3037; arXiv:gr-qc/0708.3033; A. Pound, E. Poisson, and B. G. Nickel, Phys. Rev. D 72, 124001 (2005).

[8] E. Rosenthal, Phys. Rev. D 74, 084018 (2006); 73, 044034 (2006); 72, 121503 (2005); Classical Quantum Gravity 22, S859 (2005). 